tándar tuvo su mejor desempeño cuando se emplearon los datos normalizados.

Cuando se emplearon umbrales de mayor especificidad (a manera de desencadenar el menor número de alertas), los métodos basados en la pendiente logarítmica y en el porcentaje de muestras positivas generaron la menor proporción de NCP en comparación con el resto de los algoritmos. Cuando se usaron umbrales de mayor sensibilidad se obtuvieron los resultados opuestos. En este caso, el algoritmo basado en el porcentaje de muestras positivas con un umbral $\leq 65 \%$ ofreció la mayor proporción de NCP en comparación con los otros algoritmos.

El empleo de una alerta anual con una intervención en una misma semana del año (por lo general inmediatamente antes del comienzo de la temporada de mayor transmisión) hubiera logrado prevenir $28,4 \%$ del NCP. Sin embargo, los algoritmos basados en la media semanal y los percentiles hubieran prevenido la misma proporción de casos con la mitad de las alertas anuales.

Si se hubiera empleado el percentil 85 como umbral para declarar las alertas, se habrían declarado 0,72 alertas anuales y se habría evitado $31,9 \%$ del NCP, mientras que la aplicación de umbrales basados en los percentiles 80 y 75 habría desencadenado 0,79 y 0,9 alertas anuales con $32,6 \%$ y $31,2 \%$ de NCP, respectivamente. El incremento del número de alertas anuales en $0,1-0,2$ no habría generado beneficios adicionales. En situaciones en la que el costo no constituye un factor limitante y es posible aplicar medidas preventivas anuales, el método basado en la proporción de muestras positivas puede ser el método de elección. Este método dio resultados similares o mejores que los otros métodos cuando se modelaron para realizar una alerta anual, ya que permitió identificar mejor el momento oportuno para la intervención.

Este estudio demuestra que con el empleo del percentil semanal de casos para determinar el punto de corte que define las situaciones de alerta de epidemias de malaria se pueden identificar tempranamente los períodos de mayor número de casos de esta enfermedad en Etiopía. Esto permitiría realizar intervenciones más racionales para el control de la malaria y evitaría un mayor número de casos de malaria.

La técnica comparativa expuesta en este trabajo puede emplearse para evaluar otros sistemas de alertas en otras poblaciones y con otras enfermedades. (Teklehaimanot HD, Schwartz J, Teklehaimanot A, Lipsitch M. Alert threshold algorithms and malaria epidemic detection. Emerg Infect Dis [serial on the Internet]. 2004;10(7). Hallado en: http://www.cdc.gov/ncidod/EID/vol10no7/030722.htm. Acceso el 29 de junio de 2004.)

\section{Síndrome de cansancio crónico y discapacidad laboral}

Según los Centros para el Control y la Prevención de Enfermedades (CDC) de los Estados Unidos de América, el síndrome de cansancio crónico (SCC) se define como un cansancio intenso que incapacita a los pacientes tanto física como mentalmente durante seis meses o más, que se exacerba por esfuerzos mínimos y que no se puede explicar mediante diagnósticos convencionales. Aunque en la actualidad existen varias definiciones operacionales diferentes del SCC, todas se basan en la información que aportan los pacientes. Debido a que no se cuenta con marcadores diagnósticos de laboratorio ni se han definido muestras de biopsia patognomónicas, el diagnóstico de esta enfermedad se realiza por exclusión. El SCC es una enfermedad puramente subjetiva y hasta el momento no se ha informado de tratamientos que hayan logrado mitigar la enfermedad, aunque la psicoterapia conductual cognoscitiva puede aliviar los síntomas.

El objetivo de este trabajo fue evaluar la información científica publicada sobre la detección y el control de la discapacidad en personas con SCC. Para ello se realizó una revisión sistemática en la que participó un panel multidisciplinario de expertos que evaluó los artículos publicados entre el $1 .^{\circ}$ de enero de 1988 (año en que se publicó la primera definición operacional de esta enfermedad) y el 15 de noviembre de 2001. La búsqueda bibliográfica se realizó en las bases MEDLINE, Current Contents, Cochrane Library y PsychINFO. Además se buscaron citas relacionadas con el tema en los estudios y artículos de revisión aceptados entre el $1 .^{\circ}$ de enero de 1999 y el 31 de diciembre de 2001.

Se tomaron en cuenta los estudios observacionales (prospectivos, retrospectivos y transversales) e intervensionistas, que informaran el diagnóstico de SCC según alguna de las cuatro definiciones para adultos de los CDC y que contuvieran los términos "fatigue syndrome" + "chronic" o "chronic fatigue". Solo se tomaron en cuenta los artículos publicados en inglés.

En total se identificaron 3640 estudios, de los cuales 53 cumplieron con los criterios de selección. De los estudios aceptados, 36 eran observacionales (3 210 pacientes) y en 17 se habían aplicado diferentes intervenciones (1 348 pacientes). Todos los 17 informaron de haber obtenido mejoras durante el seguimiento. Las intervenciones aplicadas fueron conductuales (cuatro estudios), psicológicas (dos estudios), con medicamentos (cinco estudios), ocupacionales (dos estudios) o combinadas (cuatro estudios). Seis estudios tenían grupos con placebo.

En total, 37 estudios informaron de la condición laboral de los pacientes y realizaron alguna me- 
dición de su discapacidad mental o física. La mayoría de los pacientes con SCC participaron en estos estudios no tenían vínculo laboral. En 22 de estos estudios también se conocía la condición laboral de los participantes de los grupos testigo.

Setenta y seis por ciento de los participantes en los estudios evaluados eran mujeres. La edad promedio de los participantes en los 48 estudios que informaron este parámetro (4 372 pacientes) fue de 38,4 años de edad (de 24,7 a 46,1 años). La duración del SCC en los 3976 pacientes de los 40 estudios que informaron este indicador fue de 5,5 años en promedio (de 1,9 a 8,5 años). La escolaridad media de los 1310 pacientes de los 14 estudios que informaron el nivel de escolaridad fue de 14,1 años (de 11,8 a 16,0 años). Las características demográficas de los participantes en los grupos testigo fueron similares a la de los pacientes con SCC.

Solo cuatro estudios longitudinales informaron de los valores encontrados antes y después de la intervención. Otros dos estudios sin intervención ofrecieron esta información. En los cuatro estudios con intervención se demostró una mejoría en la condición laboral de los pacientes, mientras que los dos estudios sin intervención mostraron un empeoramiento en este sentido.

Solo la depresión resultó asociada con el desempleo en los pacientes con SCC y solamente el tratamiento de la conducta cognitiva, la rehabilitación y los programas terapéuticos con ejercicios se asociaron con el restablecimiento de la capacidad laboral de estos pacientes. No se identificó ninguna característica específica de los pacientes con SCC que pudiera definir o servir para pronosticar su mejoría en cuanto a la capacidad laboral o funcional.

La información disponible indica que la tasa de desempleo en los pacientes con SCC es elevada. El deterioro físico y mental de estos pacientes puede ser demostrado, a pesar de que no se han validado métodos e instrumentos para ello. No se han encontrado indicadores demográficos, clínicos o psiquiátricos que permitan pronosticar la capacidad de estos pacientes para regresar a su actividad laboral.

Se requieren más investigaciones que evalúen si las características de los proveedores de servicios de salud o la experiencia laboral previa de los pacientes están relacionadas con la discapacidad de los pacientes con SFC. Por último, se necesitan nuevos estudios longitudinales con intervenciones para determinar las características iniciales asociadas con la discapacidad laboral y con intervenciones que permitan restablecer la capacidad laboral de estos pacientes. (Ross SD, Estok RP, Frame D, Stone LR, Ludensky V, Levine CB. Disability and chronic fatigue syndrome a focus on function. Arch Intern Med. 2004;164:1098-1107.)

Annual Meeting of the American Society of Tropical Medicine and Hygiene

Dates: $\quad 7-11$ November 2004

Location: Fontainebleau Hilton Hotel

Miami Beach, Florida, United States of America

The 53rd Annual Meeting of the American Society of Tropical Medicine and Hygiene (ASTMH) will include research talks, plenary sessions, scientific sessions, poster presentations, and exhibits of products and services for the tropical medicine and hygiene field. The meeting is intended for researchers, professors, government officials, public health officials, military personnel, physicians, and students.

The registration fee for the meeting, if paid by 7 October, is US $\$ 545$ for persons who are not ASTMH members and US\$ 375 for ASTMH members. After that date, the respective costs are US\$ 595 and US\$ 425. The charge for students is US\$ 175 if paid by 7 October and US\$ 225 after that date.

Information:

American Society of Tropical Medicine and Hygiene

60 Revere Drive, Suite 500 • Northbrook, Illinois 60062

United States of America

Telephone: (847) 480-9592 • Fax: (847) 480-9282

E-mail: astmh@astmh.org

Web site: http://www.astmh.org/meetings/meeting2004.html 